\title{
Minimum Energy Coding in CDMA Wireless Sensor Networks
}

\author{
C. Fischione, Member, IEEE, K. H. Johansson, Member, IEEE, A. Sangiovanni-Vincentelli, Fellow, IEEE, \\ and B. Zurita Ares
}

\begin{abstract}
A theoretical framework is proposed for accurate comparison of minimum energy coding in Coded Division Multiple Access (CDMA) Wireless Sensor Networks (WSNs). Energy consumption and reliability are analyzed for two coding schemes: Minimum Energy coding (ME), and Modified Minimum Energy coding (MME). A detailed model of consumed energy is described as function of the coding, radio transmit power, the characteristics of the transceivers, and the dynamics of the wireless channel. Since CDMA is strongly limited by multi-access interference, the system model includes all the relevant characteristics of wireless propagation. A distributed and asynchronous algorithm, which minimizes the total energy consumption by controlling the radio power, is developed. Numerical results are presented to validate the theoretical analysis and show under which conditions MME outperforms ME with respect to energy consumption and bit error rate. It is concluded that MME is more energy efficient than ME only for short codewords.
\end{abstract}

Index Terms-Wireless sensor network (WSNs), minimum energy coding, CDMA, OOK, power control, outages, parallel and distributed computation.

\section{INTRODUCTION}

W IRELESS sensor networks have the potential of dwarfing the revolution that the Internet has brought to the world of computing, entertainment, work and human interaction. They will make it possible to connect computing with the physical environment yielding the so called Physical Internet. Given the small dimensions of the sensing devices and their inaccessibility when deployed in the environment, their computing power and the energy resources are necessarily limited. Wireless communication consumes a substantial part of energy, which is related to the need of transmitting with few errors. An optimization problem arises where the energy spent in transmitting and receiving is minimized while a given probability of correct transmission is guaranteed. Source coding, radio power control and sleep disciplines are

Manuscript received February 20, 2008; revised June 20, 2008; accepted August 13, 2008. The associate editor coordinating the review of this paper and approving it for publication was K. B. Lee.

C. Fischione and K. H. Johansson are with the ACCESS Linnaeus Center, Electrical Engineering, Royal Institute of Technology, Stockholm, Sweden (e-mail: \{carlofi, kallej\}@ee.kth.se).

A. Sangiovanni-Vincentelli is with the Department of Electrical Engineering and Computer Sciences, University of California at Berkeley (e-mail: alberto@eecs.berkeley.edu).

B. Zurita Ares was with the Royal Institute of Technology, Stockholm, Sweden, when contributing to this work (e-mail: benigno.zurita@gmail.com).

A. Sangiovanni Vincentelli and C. Fischione wish to acknowledge the support of the NSF ITR CHESS and the GSRC. The work was partially funded also by the Swedish Foundation for Strategic Research and the Swedish Research Council and VINNOVA.

Part of the topics of this paper were presented at EWSN 07.

Digital Object Identifier 10.1109/TWC.2009.080239 all techniques that have been used to minimize the energy consumption of wireless sensor networks.

In this paper we focus on minimizing the energy consumption of wireless sensor networks by using low energy coding schemes. The two coding techniques analyzed here are Minimum Energy (ME) coding used in conjunction with On-Off Keying (OOK) modulation [1], [2], and the Modified Minimum Energy (MME) coding, a variant of the ME coding with sleep disciplines at the receiver [3], [4].

In ME and MME coding, information is coded in digital form so that zero-one patterns determine in large part energy consumption by the transmitter and the receiver. If we assume that the ones are the information that is actually sent while zeros correspond to no transmission, then ME optimizes energy by minimizing the number of ones present in the coded message to be transmitted. In particular, ME encodes high probability source codewords with coded codewords having a small number of ones. This simple yet powerful idea, proposed by Erin and Asada, has been extended by Prakash and Gupta [5], who have proposed ME coding along with channel coding for the case of sources with unknown statistics. Tang et al. [6] have investigated the bit error rate of ME coding and OOK modulation for both coherent and non coherent receivers in AWGN channels. Liu and Asada [7] have applied ME coding to CDMA wireless systems and reported that Multi-Access Interference (MAI) is reduced when using ME coding. Since small MAI implies fewer bit errors, ME has good reliability. ME and CDMA were considered also by Kim and Andrews [4], when they proposed the MME scheme. Since radio power plays an important role in the energy balance for transmission, it is of paramount importance to consider the joint effect of radio power control and minimum energy coding on the overall energy consumption. Zurita et al. [8] presented an analysis of ME and MME for WSNs in a slow fading propagation environment using heuristic power control and detection algorithms.

In this paper we offer a complete framework to compare and optimize the ME and MME approaches with respect to energy consumption while satisfying constraints on communication errors. To do so, we develop

- an accurate characterization of the energy spent for coding, transmitting and receiving (taking into account radio power, and average number of radio module startups) including a detailed model of the MAI and wireless channel with path loss, slow and fast fading (presented in Section III);

- a decentralized radio power control algorithm, which 


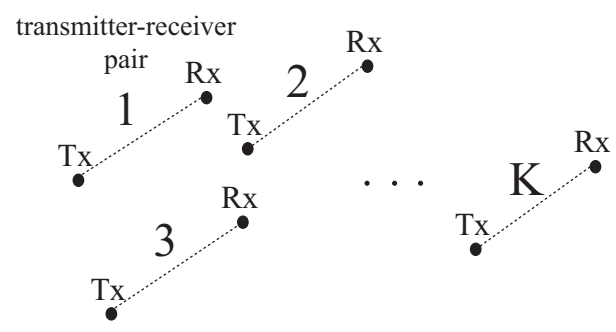

Fig. 1. System scenario: $K$ asynchronous pairs of nodes are simultaneously transmitting.

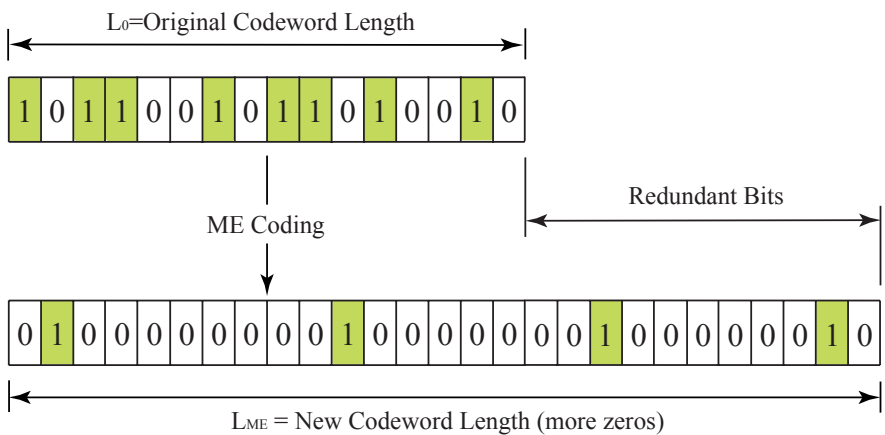

Fig. 2. Mapping of the source codewords into the codewords of the Minimum Energy Coding (from [7])

minimizes the total energy spent for transmission and reception using the models above (Section IV);

- an optimal decision threshold for OOK detection, as function of the ME and MME coding schemes and radio powers (Section V);

- a characterization of the bit error probabilities of ME and MME coding when the radio power control algorithm is deployed (Section VI).

Comparing our approach to existing contributions [1], [2] and [4] - [7], we are able to provide a characterization of total energy consumption with respect to all the parameters of a complete system scenario, namely: the energy due to ME, MME, CDMA, the wireless channel, and the transceiver. As a consequence of this more detailed study, our analysis leads to a different result from the literature when comparing $\mathrm{ME}$ and MME as shown in the numerical results presented in Section VII.

\section{SySTEM DESCRIPTION}

Consider a scenario where there are $K$ transmitter-receiver pairs of nodes (see Fig. 1). Data sensed by a node is at first coded either with ME or with MME coding.

With ME coding [1], [2], each codeword having large probability is mapped into a new codeword having less number of one (or high) bits. Denote with $L_{0}$ the length of the source codeword, and with $L_{M E}$ the length of the ME codeword, where $L_{0} \leq L_{M E}$. The extra bits added are the redundant bits needed for channel coding (see Fig. 2). Let $\alpha_{\mathrm{ME}}$ be the probability of having high bits in an ME codeword.

MME coding [4] exploits a structure of the codeword that allows the receiver to go in a sleep state, where the radio electronic circuitry is switched off [3]. With MME, the ME

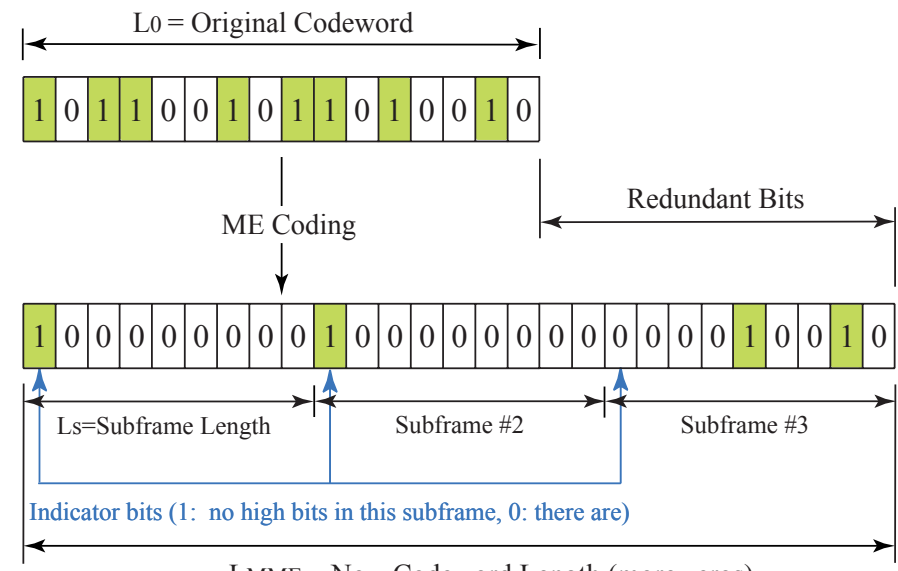

LMME $=$ New Codeword Length (more zeros)

$\mathrm{Ns}_{\mathrm{s}}=$ number of subframes

Fig. 3. MME codeword. The original codeword is mapped into a MME codeword partitioned in sub-frames. Each sub-frame starts with an indicator bit (from [4]).

codewords are partitioned into $N_{s}$ sub-frames of length $L_{s}$, where each sub-frame starts with an indicator bit $b_{\text {ind }}$ (see Fig. 3). When $b_{\text {ind }}$ is a one (high bit), it indicates that there are no high bits in that sub-frame, so there is no need for decoding, and the receiver can go to the sleep state. Conversely, if $b_{\text {ind }}$ is a zero (low bit), it indicates that there are high bits in the sub-frame, so the decoding operation must be performed, and the receiver cannot go to sleep.

The ME or MME coded bits are then handled by an OOK modulator: only bits having value one (high bits) are DS-CDMA processed and transmitted. An asynchronous DSCDMA wireless access scheme is considered, where the same fixed bandwidth $W$ is allocated to each transmitter-receiver pair. The processing gain is denoted with $G=T_{b} / T_{c}$, where $T_{b}$ is the bit interval, and $T_{c}$ is the chip interval. The transmitted signal, after being attenuated by the wireless channel, is received corrupted by an additive Gaussian noise and MAI caused by other transmitting nodes. The output of the coherent correlation receiver of link $i$ can be expressed as [9]

$$
Z_{i}(t)=D_{i}(t)+I_{i}(t)+N_{g}(t)
$$

where $D_{i}(t)$ is the signal bearing the information for the pair $i, I_{i}(t)$ is the interference due to the presence of multiple transmitting nodes (causing MAI) and $N_{g}(t)$ is the AWGN noise, which is modeled as a Gaussian random variable with zero mean and variance $N_{0} T_{b} / 4$. In particular, it can be proved that [9]

$$
D_{i}(t)=\nu_{i}(t) \sqrt{\frac{h_{i, i}(t) P_{i}}{2}} T_{b} \triangleq \nu_{i}(t) \mu_{Z_{i}}(t),
$$

and that the variance of the MAI plus the AWGN, on a bit time scale and conditioned to the distribution of the high bits and the wireless channel, is

$$
\frac{T_{b}^{2}}{6 G} \sum_{\substack{j=1 \\ j \neq i}}^{K} \nu_{j}(t) h_{j i}(t) P_{j}+\frac{T_{b}}{4} N_{0} \triangleq \sigma_{Z_{i}}^{2}(t) .
$$




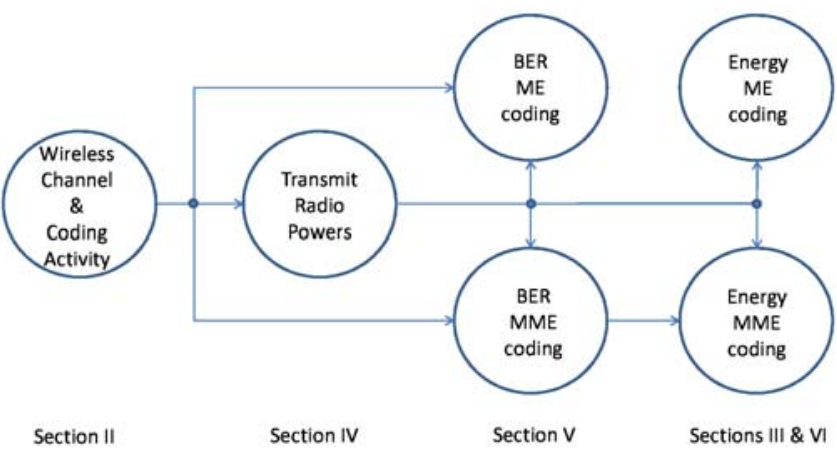

Fig. 4. Relation among the system parameters (wireless channel, coding, transmit radio power) and the bit error rate (BER) and the energy consumption of $\mathrm{ME}$ and MME.

In (2) and (3), $P_{i}, i=1, \ldots, K$, denotes the radio power of the sender node in link $i$. We introduce the vector $\mathbf{P}=\left[P_{1}, \ldots, P_{i}, \ldots, P_{K}\right]^{T}$. The term $\nu_{j}(t)$ is a binary random variable abstracting the transmission of a high bit $\left(\nu_{j}(t)=1\right)$ or low bits $\left(\nu_{j}(t)=0\right)$, with probability mass function $\operatorname{Pr}\left[\nu_{j}(t)=1\right]=a$ and $\operatorname{Pr}\left[\nu_{j}(t)=0\right]=1-a$, respectively. In particular, $a=\alpha_{\mathrm{ME}}$ for $\mathrm{ME}$ coding, whereas $a=\alpha_{\mathrm{MME}}+N_{s} /\left(N_{s} L_{s}\right)=\alpha_{\mathrm{MME}}+1 / L_{s}$ for MME coding, because indicator bits have been added to the MME codeword with respect to the ME codeword (recall Fig. 2 and Fig. 3). Throughout the paper, we assume that $\alpha_{\mathrm{ME}}=\alpha_{\mathrm{MME}}$. Let vector $\boldsymbol{\nu}(t)=\left[\nu_{1}(t), \ldots, \nu_{i}(t), \ldots \nu_{K}(t)\right]^{T}$ denote the nodes' coding activity.

In (2) and (3), the wireless channel coefficient associated to the path from the transmitter of link $j$ to the receiver of link $i$ is $h_{j i}(t)=l_{j i} r_{j i}(t) \exp \xi_{j i}(t)$ where $l_{j i}$ is the path loss, which is dependent on the distance and propagation environment [10]. We consider the Nakagami distribution for the fast fading, with correlation 1 and parameter $m$, so $r_{j}(t)$ has a gamma distribution having average $\mu_{r_{i}}$ and correlation $\rho_{r_{i}}$ [10]. The term $\exp \xi_{j i}(t)$ is the shadow fading component, with $\xi_{j i}(t)$ being a Gaussian random variable having zero average and standard deviation $\sigma_{\xi_{j i}}$. We introduce the vector $\mathbf{h}_{i}(t)=\left[h_{1, i}(t), \ldots, h_{i, i}(t), \ldots h_{K, i}(t)\right]^{T}$ to denote the wireless channel coefficients seen by the receiver of the pair $i$.

\section{ENERGY MODEL}

Here we present an original characterization of the energy spent to transmit information using $\mathrm{ME}$ and $\mathrm{MME}$ as function of the coding activity, the transmit radio powers, the wireless channel, and the hardware platform. The relation among these system parameters with the bit error rate and the energy consumption of ME and MME is summarized in Fig. 4.

\section{A. ME Coding}

Consider link $i$ between a pair of transmitter and receiver nodes. The energy consumption per ME codeword spent over the link can be expressed as follows:

$$
\begin{aligned}
E_{i}^{(\mathrm{ME})}= & E_{i}^{(\mathrm{tx})}+E_{i}^{(\mathrm{rx})} \\
= & P^{(\mathrm{tx}, \mathrm{ckt})}\left[T^{(\mathrm{tx}, \mathrm{ME})}+T_{s}\right]+\alpha_{\mathrm{ME}} f\left(P_{i}\right) T^{(\mathrm{tx}, \mathrm{ME})} \\
& +P^{(\mathrm{rx}, \mathrm{ckt})}\left[T^{(\mathrm{rx}, \mathrm{ME})}+T_{s}\right]
\end{aligned}
$$

where $E_{i}^{(\mathrm{tx})}$ and $E_{i}^{(\mathrm{rx})}$ are the average energy consumption of a node while transmitting and receiving, respectively. The power consumption of the electronic circuits, while transmitting and processing a codeword, is denoted with $P^{\text {(tx ,ckt) }}$, and while receiving is denoted with $P^{\text {(rx,ckt) }}$. Note that $P^{\text {(tx,ckt) }}$ and $P^{\text {(rx,ckt) }}$ do not include the radio power, which is $P_{i}$. The function $f\left(P_{i}\right)$ accounts for the energy spent by the radio module to transmit at radio power $P_{i} . f\left(P_{i}\right)$ is an increasing function of $P_{i}$, since the higher is the radio power transmitted, the higher is the energy drained by the transmit antenna. $T^{\text {(tx }, \mathrm{ME})}$ is the transmitter activity time per ME codeword, and $T^{\text {(rx, ME) }}$ is the receiver activity time per codeword; finally, $T_{s}$ is the start up time of the radio transceiver, i.e., the time employed to go from the sleep state to the active state.

\section{B. MME Coding}

By adopting the same parameter definition used in (4), we model the energy consumption per MME codeword as follows:

$$
\begin{aligned}
E_{i}^{(\mathrm{MME})}= & E_{i}^{(\mathrm{tx})}+E_{i}^{(\mathrm{rx})} \\
= & P^{(\mathrm{tx}, \mathrm{ckt})}\left[T^{(\mathrm{tx}, \mathrm{MME})}+T_{s}\right] \\
& +\alpha_{\mathrm{MME}} f\left(P_{i}\right) T^{(\mathrm{tx}, \mathrm{MME})}+P^{(\mathrm{rx}, \mathrm{ckt})} T^{(\mathrm{rx}, \mathrm{MME})} \\
& +\left[P^{(\mathrm{rx}, \mathrm{ckt})}+P^{(\mathrm{rx}, \mathrm{ckt}, \mathrm{st})}\right]\left(N_{i}+1\right) T_{s}
\end{aligned}
$$

In (5), $T^{(\mathrm{tx}, \mathrm{MME})}$ is the transmitter activity time per MME codeword, and $T^{(\mathrm{rx}, \mathrm{MME})}$ is the receiver activity time per codeword. We will see later that $T^{(\mathrm{rx}, \mathrm{MME})}$ depends on the radio powers. $P^{\text {(rx,ckt,st) }}$ is the extra energy overhead spent in the start-up phase. In (5), we have introduced the average number of times, denoted with $N_{i}$, that the receiver has to awake from the sleep state. This term, as $T^{\text {(rx, MME) }}$ depends on the bit error rate, and hence on the radio powers. It plays a fundamental role when evaluating MME energy consumption: each time the radio receiver module is turned on, it spends an amount of energy given by $P^{(\mathrm{rx}, \mathrm{ckt})} T_{s}$. The term $\left[P^{\text {(rx,ckt })}+P^{(\mathrm{rx}, \mathrm{ckt}, \mathrm{st})}\right]\left(N_{i}+1\right) T_{s}$ may be an important piece of energy, because $P^{\text {(rx,ckt) }}$ is the largest term among the power components at the receiver (it is comparable with the largest value of $f\left(P_{i}\right) T^{(\mathrm{tx}, \mathrm{MME})}$ in off-the-shelf nodes [11] [12]), $T_{s}$ is not negligible, and neither is $N_{i} . f\left(P_{i}\right)$ and $N_{i}$ have not been included in the energy model proposed in [4]. The consequence of taking all these parameters into account will lead to the conclusion that MME offers adequate performance improvement with respect to $\mathrm{ME}$ only in some but not all cases, as we shall see later.

In the following sections, we investigate the components that concur to the energy consumption of $\mathrm{ME}$ and $\mathrm{MME}$ coding, namely, the energy needed for radio transmission, and the bit error rate, which determines the number of starts-up of the radio module.

\section{Optimal Transmit Radio Power}

In this section, we propose an algorithm for minimizing the total energy consumption by optimally selecting the radio powers $P_{i}$. Radio powers must be allocated to ensure a given quality of the received signal. The Signal to Interference plus Noise Ratio (SINR) is a typical quality measure. Consider link 
$i$ between a pair of transmitter and a receiver nodes. The SINR is defined as

$$
\operatorname{SINR}_{i}(t)=\frac{\nu_{i}(t) \mu_{Z_{i}}^{2}(t)}{\sigma_{Z_{i}}^{2}(t)}
$$

Note that the SINR is a random variable, since it depends on the wireless channel coefficients $\mathbf{h}_{i}(t)$, as well as on the coding $\nu_{i}(t)$. Moreover, it is function of the transmit powers $\mathbf{P}$. When $\nu_{i}(t)=0$, the SINR is zero.

To minimize the total energy consumption of the overall system, we propose an optimization problem whose objective function is the sum of the energy to transmit and receive, while the constraints are expressed in terms of outage probability of the SINR:

$$
\begin{array}{ll}
\mathcal{P}: & \min _{\mathbf{P}} \sum_{i=1}^{K} E_{i}(\mathbf{P}) \\
\text { s.t. } & \operatorname{Pr}\left[\operatorname{SINR}_{i}(t) \leq \gamma \mid \nu_{i}(t)=1\right] \leq \bar{P}_{\text {out }}, \\
& \\
& i=1, \ldots, K .
\end{array}
$$

In Problem $\mathcal{P}, E_{i}(\mathbf{P})$ is the total energy consumption, and it is given by (4) for ME coding, and by (5) for MME coding. $\gamma$ is the SINR threshold under which outages occur. Solving the optimization problem ensures that the outage probabilities remain below $\bar{P}_{\text {out }}$. We assume that Problem $\mathcal{P}$ is feasible throughout the paper. From a physical point of view, feasibility means that all nodes can transmit with a certain radio power while satisfying the outage constraint.

To solve Problem $\mathcal{P}$, the constraints related to the outage have to be modeled. Note that the outage probability depends on the distribution of the SINR, which, in turn, depends on the wireless channel $\mathbf{h}_{i}(t)$ and the distribution of high bits $\boldsymbol{\nu}(t)$ (i.e., the coding). Since the distribution of the SINR is unknown, we make use of an approximation.

We note that $\mu_{Z_{i}}^{2}(t)$ in the numerator of the SINR (6) is a product of a gamma random variable, $r_{i i}(t)$, and a log-normal one, $\exp \xi_{i i}(t)$. Then, as proposed in [10], this product can be well approximated with a log-normal random variable:

$$
\mu_{Z_{i}}^{2}(t)=\frac{T_{b}^{2}}{2} P_{i} l_{i i} r_{i i}(t) \exp \xi_{i i}(t) \approx \exp X_{i}(t) .
$$

where $X_{i}(t)$ is a Gaussian random variable having average and standard deviation, respectively $\mu_{X_{i}}=\ln \left(P_{i} l_{i i} T_{b}^{2}\right)-\ln 2+$ $\psi(m)-\ln m$ and $\sigma_{X_{i}}^{2}=\zeta(2, m)+\sigma_{\xi_{i i}}^{2}$, where $\psi(m)$ is Euler's psi function, and $\zeta(2, m)$ is Riemann's zeta function.

The denominator of the SINR is sum of log-normal random variables weighted by gamma and binary random variables. Therefore, following the same approach as in [13], the Wilkinson's moment-matching method can be applied and the denominator is well approximated with a log-normal random variable $\sigma_{Z_{i}}^{2}(t) \approx \exp \left(Y_{i}(t)\right)$, where $Y_{i}(t)$ is a Gaussian random variable having average and standard deviation obtained by matching the first and second order moments of $\sigma_{Z_{i}}^{2}(t)$ :

$$
\begin{aligned}
& \mu_{Y_{i}}=2 \ln \mathbb{E}_{\boldsymbol{\nu}(t), \mathbf{h}_{i}(t)}\left[\sigma_{Z_{i}}^{2}(t)\right]-\frac{1}{2} \ln \mathbb{E}_{\boldsymbol{\nu}(t), \mathbf{h}_{i}(t)}\left[\sigma_{Z_{i}}^{4}(t)\right], \\
& \sigma_{Y_{i}}^{2}=\ln \mathbb{E}_{\boldsymbol{\nu}(t), \mathbf{h}_{i}(t)}\left[\sigma_{Z_{i}}^{4}(t)\right]-2 \ln \mathbb{E}_{\boldsymbol{\nu}(t), \mathbf{h}_{i}(t)}\left[\sigma_{Z_{i}}^{2}(t)\right] .
\end{aligned}
$$

The expressions of $\mathbb{E}_{\boldsymbol{\nu}(t), \mathbf{h}_{i}(t)}\left[\sigma_{Z_{i}}^{2}(t)\right]$ and $\mathbb{E}_{\boldsymbol{\nu}(t), \mathbf{h}_{i}(t)}\left[\sigma_{Z_{i}}^{4}(t)\right]$ can be easily derived by applying the linear and distributive properties of the statistical expectation, and remembering that the vectors $\mathbf{h}_{i}(t)$ and $\boldsymbol{\nu}(t)$ are statistically independent [8].

Using the previous approximations, the outage probability can be computed while taking into account the wireless coefficients, the transmission powers, and the distribution of high bits. It follows that

$$
\begin{aligned}
& \operatorname{Pr}\left[\operatorname{SINR}_{i}(t) \leq \gamma \mid \nu_{i}(t)=1\right] \approx \operatorname{Pr}\left[\exp \left(X_{i}(t)-Y_{i}(t)\right) \leq \gamma\right] \\
& \quad=1-Q\left(\frac{\ln \gamma-\mu_{X_{i}}+\mu_{Y_{i}}}{\sqrt{\sigma_{X_{i}}^{2}+\sigma_{Y_{i}}^{2}}}\right)
\end{aligned}
$$

where $Q(x)=1 / \sqrt{2 \pi} \int_{x}^{\infty} e^{-t^{2} / 2} d t$ is the complementary standard Gaussian distribution. Expression (9) can be used to rewrite the constraints in Problem $\mathcal{P}$, which becomes:

$$
\begin{array}{ll}
\mathcal{P}: & \min _{\mathbf{P}} \sum_{i=1}^{K} E_{i}(\mathbf{P}) \\
\text { s.t. } & P_{i} \geq g_{i}(\mathbf{P}), i=1, \ldots, K
\end{array}
$$

where $g_{i}(\mathbf{P})=\gamma \exp \left(-\mu_{X_{i}}+\ln P_{i}+\mu_{Y_{i}}-q_{i} \sqrt{\sigma_{X_{i}}^{2}+\sigma_{Y_{i}}^{2}}\right)$ and $q_{i}=Q^{-1}\left(1-\bar{P}_{\text {out }}\right)$.

Problem $\mathcal{P}$ is a centralized problem, in the sense that a central entity needs to collect information related to all radio link coefficients, compute the solution, and finally dispatch the solution to all other transmitting nodes. A centralized implementation has obvious disadvantages in terms of communication resources, delays and robustness. Nevertheless, by following the method proposed in [14], Problem $\mathcal{P}$ can be solved using a distributed strategy. First, it is possible to show that $g_{i}(\mathbf{P})$ does not depend on $P_{i}$, because neither $\mu_{X_{i}}-\ln P_{i}, \sigma_{X_{i}}, \mu_{Y_{i}}$, nor $\sigma_{Y_{i}}$ depend on $P_{i}$. Furthermore, $g_{i}(\mathbf{P})$ is monotonically non-decreasing with $\mathbf{P}$. From these properties, we have the following result:

Theorem 1: Let $E_{i}(\mathbf{P}): \mathbb{R}^{K} \rightarrow \mathbb{R}$ be an increasing function of $\mathbf{P}$. Then, Problem $\mathcal{P}$ admits a unique optimal solution $\mathbf{P}^{*}$, such that $P_{i}^{*}=g_{i}\left(\mathbf{P}^{*}\right)$. in [14].

Proof: The proof is similar to the proof of Theorem 1 This theorem suggests solving Problem $\mathcal{P}$ just by looking at the solution of the system of non-linear equations given by the constraints. Since $g_{i}(\mathbf{P})$ is a non-decreasing function, it is also a contraction mappings in $\mathbf{P}$. The component solution method, as defined in [15, Pag.181-191], can be applied to solve Problem $\mathcal{P}$ in a fully distributed and asynchronous manner using the following algorithm:

$$
P_{i}(n):=g_{i}(\mathbf{P}(n-1)),
$$

where $n$ is a local iteration time. The algorithm is stopped when $\left|P_{i}(n)-P_{i}(n-1)\right| \leq \varepsilon$, where $\varepsilon$ denotes the precision of the solution. The algorithm is fully distributed, because at receiver node of link $i$ we need not know the transmit powers of all nodes, but only the statistical moments $\mu_{X_{i}}, \sigma_{X_{i}}$, $\mu_{Y_{i}}$, and $\sigma_{Y_{i}}$, which can be easily estimated locally through sample averages. Algorithm (10) converges exponentially to the optimal solution [15]. The numerical results of Section VII show that convergence is fast; in the example described in Section VII, convergence was achieved in less than 5 iterations. 
In the sequel, we assume that the radio powers are computed by solving Problem $\mathcal{P}$. We investigate the bit error probabilities of ME and MME, and how these probabilities relate to the total energy consumption.

\section{Bit ERror Probability}

The derivation of the BER using the decision variable (1) distinguishes two cases of error: the decision variable is decoded as a low bit, when a high bit was transmitted; or the decision variable is decoded as a high bit when a low bit was transmitted. We denote these probabilities with $e_{i \mid 0, \mathbf{h}_{i}(t), \boldsymbol{\nu}(t)}$ and $e_{i \mid 1, \mathbf{h}_{i}(t), \nu(t)}$, respectively, where:

$$
\begin{array}{rr}
e_{i \mid 0, \mathbf{h}_{i}(t), \boldsymbol{\nu}(t)}= & \operatorname{Pr}\left[Z_{i}(t) \geq \delta_{i} \mid \nu_{i}(t)=0, \mathbf{h}_{i}(t), \boldsymbol{\nu}(t)\right] \\
= & Q\left(\frac{\delta_{i}}{\sigma_{Z_{i}}(t)}\right), \\
e_{i \mid 1, \mathbf{h}_{i}(t), \boldsymbol{\nu}(t)}= & \operatorname{Pr}\left[Z_{i}(t)<\delta_{i} \mid \nu_{i}(t)=1, \mathbf{h}_{i}(t), \boldsymbol{\nu}(t)\right] \\
= & Q\left(\frac{\mu_{Z_{i}}(t)-\delta_{i}}{\sigma_{Z_{i}}(t)}\right),
\end{array}
$$

where $\delta_{i}$ is the decision threshold for the variable $Z_{i}(t)$ and $\mu_{Z_{i}}(t)$ and $\sigma_{Z_{i}}^{2}(t)$ have been defined in (8) and (3), respectively. The probabilities in (11) and (12) are computed under the obvious assumption that the decision threshold $\delta_{i}$ must lie in the interval $\left[0, \mu_{Z_{i}}(t)\right]$, and adopting the usual standard Gaussian approximation [10], where $Z_{i}(t)$ is modelled as a Gaussian random variable conditioned to the distribution of the channel coefficients and coding. Specifically, it is assumed that $Z_{i}(t) \sim \mathcal{N}\left(\nu_{i}(t) \mu_{Z_{i}}(t), \sigma_{Z_{i}}(t)\right)$.

The bit error probability, conditioned to the channel coefficients and coding, is

$$
\begin{aligned}
\Phi_{i \mid \mathbf{h}_{i}(t), \boldsymbol{\nu}(t)}\left(\delta_{i}\right)= & \operatorname{Pr}\left[\nu_{i}(t)=0\right] e_{i \mid 0, \mathbf{h}_{i}(t), \boldsymbol{\nu}(t)}\left(\delta_{i}\right) \\
& +\operatorname{Pr}\left[\nu_{i}(t)=1\right] e_{i \mid 1, \mathbf{h}_{i}(t), \boldsymbol{\nu}(t)}\left(\delta_{i}\right) \\
= & (1-a) Q\left(\frac{\delta_{i}}{\sigma_{Z_{i}}(t)}\right)+a Q\left(\frac{\mu_{Z_{i}}(t)-\delta_{i}}{\sigma_{Z_{i}}(t)}\right) .
\end{aligned}
$$

This expression could be minimized with respect to $\delta_{i}$. Indeed, (13) is convex in $\delta_{i}$, because $Q(x)$ is convex for $x \geq 0$, whereas the arguments of the first and second $Q$ are concave, and a convex function of a concave function is convex [16]. We can analytically solve for the optimal solution:

$$
\delta_{i}^{*}=\frac{\mu_{Z_{i}}(t)}{2}-\frac{\sigma_{Z_{i}}^{2}(t)}{2 \mu_{Z_{i}}(t)} \ln \frac{a}{1-a},
$$

where the optimal threshold is dependent on the instantaneous values of the channel coefficients $\mathbf{h}_{i}(t)$ via $\mu_{Z_{i}}(t)$ and $\sigma_{Z_{i}}(t)$. However, there are at least two reasons that prevent using (14): first, for every bit time, each node should be able to detect if other nodes are transmitting (i.e. instantaneous global coding activity knowledge is required); second, each receiver node should be equipped with a channel estimator that provides the vector $\mathbf{h}_{i}(t)$ at each bit time instant (i.e. global instantaneous wireless channel estimation for each interfering node is required). Implementing these tasks on local nodes is prohibitive, since they have reduced computing resources. An alternative approach is based on taking the average of the BER with respect to the channel coefficient and coding, and then minimizing the resulting expression. With this approach, the optimal threshold depends only on the averages of the MAI, which is simple to compute, as we discuss below.
By averaging (13) with respect to $\mathbf{h}_{i}(t)$ and $\boldsymbol{\nu}(t)$ we obtain

$$
\begin{aligned}
\Phi_{i}\left(\delta_{i}\right)= & \mathbb{E}_{\boldsymbol{\nu}(t), \mathbf{h}_{i}(t)}\left[(1-a) Q\left(\frac{\delta_{i}}{\sigma_{Z_{i}}(t)}\right)\right. \\
& \left.+a Q\left(\frac{\mu_{Z_{i}}(t)-\delta_{i}}{\sigma_{Z_{i}}(t)}\right)\right] .
\end{aligned}
$$

Minimizing (15) with respect to $\delta_{i}$ is difficult, because the function is non linear and no closed-form is available for $\Phi_{i}\left(\delta_{i}\right)$. Nevertheless, numerical techniques can be applied. First, we use the Stirling approximation to compute the average of a $Q$ function: given a log-normal random variable $\zeta_{i b}$, having average $\mu_{\zeta_{i b}}$ and variance $\sigma_{\zeta_{i b}}^{2}$, the following approximation follows [10]:

$$
\begin{aligned}
\mathbb{E}\left[Q\left(\zeta_{i b}\right)\right] \approx & \frac{2}{3} Q\left(\mu_{\zeta_{i b}}\right)+\frac{1}{6} Q\left(\mu_{\zeta_{i b}}+\sqrt{3} \sigma_{\zeta_{i b}}\right) \\
& +\frac{1}{6} Q\left(\mu_{\zeta_{i b}}-\sqrt{3} \sigma_{\zeta_{i b}}\right) \triangleq F\left(\zeta_{i b}\right) .
\end{aligned}
$$

Now, define the random variables

$$
\begin{aligned}
\zeta_{i 0}\left(\delta_{i}\right) & =\frac{\delta_{i}}{\sigma_{Z_{i}}(t)} \approx \delta_{i} \exp \left(-\frac{Y_{i}(t)}{2}\right), \\
\zeta_{i 1}\left(\delta_{i}\right) & =\frac{\mu_{Z_{i}}(t)-\delta_{i}}{\sigma_{Z_{i}}(t)} \approx\left[\exp \left(\frac{X_{i}(t)}{2}\right)-\delta_{i}\right] \exp \left(-\frac{Y_{i}(t)}{2}\right) .
\end{aligned}
$$

Computing the average and standard deviation of a log-normal random variable from the natural logarithm of the variable, we have:

$$
\begin{aligned}
& \mu_{\zeta_{i 0}}\left(\delta_{i}\right)=\delta_{i} \exp \left(-\frac{1}{2} \mu_{Y_{i}}+\frac{1}{8} \sigma_{Y_{i}}^{2}\right), \\
& \sigma_{\zeta_{i 0}}^{2}\left(\delta_{i}\right)=\delta_{i}^{2} \exp \left(-\mu_{Y_{i}}+\frac{1}{2} \sigma_{Y_{i}}^{2}\right)\left[1-\exp \left(-\frac{1}{4} \sigma_{Y_{i}}^{2}\right)\right],
\end{aligned}
$$

and

$$
\begin{aligned}
\mu_{\zeta_{i 1}}\left(\delta_{i}\right)= & {\left[\exp \left(\frac{1}{2} \mu_{X_{i}}+\frac{1}{8} \sigma_{X_{i}}^{2}\right)-\delta_{i}\right] } \\
& \cdot \exp \left(-\frac{1}{2} \mu_{Y_{i}}+\frac{1}{8} \sigma_{Y_{i}}^{2}\right), \\
\sigma_{\zeta_{i 1}}^{2}\left(\delta_{i}\right)= & {\left[\exp \left(\mu_{X_{i}}+\frac{1}{2} \sigma_{X_{i}}^{2}\right)+\delta_{i}^{2}\right.} \\
& \left.-2 \delta_{i} \exp \left(\frac{1}{2} \mu_{X_{i}}+\frac{1}{8} \sigma_{X_{i}}^{2}\right)\right] \exp \left(-\mu_{Y_{i}}+\frac{1}{2} \sigma_{Y_{i}}^{2}\right) \\
& -\left[\exp \left(\mu_{X_{i}}+\frac{1}{4} \sigma_{X_{i}}^{2}\right)+\delta_{i}^{2}\right. \\
& \left.-2 \delta_{i} \exp \left(\frac{1}{2} \mu_{X_{i}}+\frac{1}{8} \sigma_{X_{i}}^{2}\right)\right] \exp \left(-\mu_{Y_{i}}+\frac{1}{4} \sigma_{Y_{i}}^{2}\right) .
\end{aligned}
$$

Using these expressions, together with (15) and (16), we obtain

$$
\begin{aligned}
\Phi_{i}\left(\delta_{i}\right) \approx & \frac{2}{3}\left[(1-a) Q\left(\mu_{\zeta_{i 0}}\left(\delta_{i}\right)\right)+a Q\left(\mu_{\zeta_{i 1}}\left(\delta_{i}\right)\right)\right] \\
& +\frac{1}{6}\left[(1-a) Q\left(\mu_{\zeta_{i 0}}\left(\delta_{i}\right)+\sqrt{3} \sigma_{\zeta_{i 0}}\left(\delta_{i}\right)\right)\right. \\
& +a Q\left(\mu_{\zeta_{i 1}}\left(\delta_{i}\right)+\sqrt{3} \sigma_{\zeta_{i 1}}\left(\delta_{i}\right)\right) \\
& +(1-a) Q\left(\mu_{\zeta_{i 0}}\left(\delta_{i}\right)-\sqrt{3} \sigma_{\zeta_{i 0}}\left(\delta_{i}\right)\right) \\
& \left.+a Q\left(\mu_{\zeta_{i 1}}\left(\delta_{i}\right)-\sqrt{3} \sigma_{\zeta_{i 1}}\left(\delta_{i}\right)\right)\right]
\end{aligned}
$$

Simple optimization algorithms such as the steepest descent or the bisection algorithms [16] can be applied to compute the 
minimum of $\Phi_{i}\left(\delta_{i}\right)$. Let $\delta_{i}^{*}$ be the value of $\delta_{i}$ that minimizes $\Phi_{i}\left(\delta_{i}\right)$. Finally, denote the average values of (11) and (12) computed in $\delta_{i}^{*}$ as

$$
\begin{aligned}
& e_{i \mid 0}=\mathbb{E} e_{i \mid 0, \mathbf{h}_{i}(t), \boldsymbol{\nu}(t)}=F\left(\zeta_{i 0}\left(\delta_{i}^{*}\right)\right), \\
& e_{i \mid 1}=\mathbb{E} e_{i \mid 1, \mathbf{h}_{i}(t), \boldsymbol{\nu}(t)}=F\left(\zeta_{i 1}\left(\delta_{i}^{*}\right)\right) .
\end{aligned}
$$

These expressions are used next to derive the bit error probability for the ME and MME coding.

\section{A. Bit Error Probability in ME Coding}

The BER in the ME case, denoted with $\Phi_{i}^{(\mathrm{ME})}$, can be easily computed by using (17) and $\delta_{i}^{*}$, where $\alpha$ takes the value $\alpha_{\mathrm{ME}}$.

\section{B. Bit Error Probability in MME Coding}

An analysis on the MME performance regarding its BER demands a careful study which takes into account the special nature of the MME codeword, as we see next.

We compute the average BER of the MME coding as the ratio between the average number of erroneous bits per MME codeword and the codeword length:

$$
\Phi_{i}^{(\mathrm{MME})}=\frac{\bar{n}_{i, s f} N_{s}}{L_{\mathrm{MME}}}
$$

where $N_{s}$ is the number of sub-frames per codeword and $\bar{n}_{i, s f}$ is the average number of erroneous bits in a sub-frame transmitted over link $i$

$$
\bar{n}_{i, s f}=\sum_{n=1}^{L_{s}-1} n \Psi_{i}(n),
$$

where $\Psi_{i}(n)$ stands for the probability of having $n$ errors in the sub-frame. To characterize $\Psi_{i}(n)$, we need the following definitions: the event $\mathcal{A}_{i}(n)$ happens when the indicator bit $b_{\text {ind }}$ was transmitted and there are $n$ decoding errors in the following sub-frame; the event $\mathcal{B}_{i}(n)$ happens when there are $n$ high bits in the sub-frame. Then

$$
\operatorname{Pr}\left[\mathcal{A}_{i}(n) \mid b_{\text {ind }}\right]=\left(\begin{array}{c}
L_{s}-1 \\
n
\end{array}\right) e_{i \mid b_{\text {ind }}}^{n}\left(1-e_{i \mid b_{\text {ind }}}\right)^{L s-1-n}
$$

and

$$
\operatorname{Pr}\left[\mathcal{B}_{i}(n)\right]=\left(\begin{array}{c}
L_{s}-1 \\
n
\end{array}\right) \alpha_{\mathrm{MME}}^{n}\left(1-\alpha_{\mathrm{MME}}\right)^{L s-1-n} .
$$

Also,

$$
\begin{aligned}
& \operatorname{Pr}\left[b_{\text {ind }}=0\right]=1-\left(1-\alpha_{\mathrm{MME}}\right)^{L_{s}-1}, \\
& \operatorname{Pr}\left[b_{\text {ind }}=1\right]=\left(1-\alpha_{\mathrm{MME}}\right)^{L_{s}-1} .
\end{aligned}
$$

Then, we have the following result

Proposition 1: The probability of detecting $n$ errors in a sub-frame is

$$
\begin{aligned}
\Psi_{i}(n)= & \operatorname{Pr}\left[b_{\text {ind }}=1\right] e_{i \mid 1} \operatorname{Pr}\left[\mathcal{A}_{i}(n) \mid b_{\text {ind }}=1\right] \\
& +\operatorname{Pr}\left[b_{\text {ind }}=0\right]\left\{\left(1-e_{i \mid 0}\right) \operatorname{Pr}\left[\mathcal{A}_{i}(n) \mid b_{\text {ind }}=0\right]\right. \\
& \left.+e_{i \mid 0} \operatorname{Pr}\left[\mathcal{B}_{i}(n)\right]\right\}
\end{aligned}
$$

Proof: Recalling that each sub-frame starts with an indicator bit, $\Psi_{i}(n)$ can be computed considering three cases:

1) The indicator bit is a one and the receiver erroneously decodes the indicator bit as a zero, with catastrophic consequences on the decoding of the sub-frame. The receiver decodes the bits of the sub-frame finding $n$ errors with probability $\operatorname{Pr}\left[\mathcal{A}_{i}(n) \mid b_{\text {ind }}=1\right]$.

2) The indicator bit is zero and the receiver performs decoding correctly. Then the receiver detects the bits of the sub-frame, finding $n$ bits in error with probability $\operatorname{Pr}\left[\mathcal{A}_{i}(n) \mid b_{\text {ind }}=0\right]$.

3) The indicator bit is zero and the receiver erroneously interprets it as a one. Then the receiver catastrophically considers all the following bits as if it were zero, making $n$ errors with probability $\operatorname{Pr}\left[\mathcal{B}_{i}(n)\right]$.

By summing up the probabilities of all the cases above, we obtain (20), which concludes the proof.

Finally, the BER of MME is given by (18), (19), and (20).

\section{ENERGY CONSUMPTION}

Here we put together the analysis presented in Sections IV and $V$ to characterize the energy consumption of the $\mathrm{ME}$ and MME schema accurately.

\section{A. ME Coding}

The energy consumption of the ME coding scheme is defined as the average of the energy consumption of all the sensor nodes:

$$
E^{(\mathrm{ME})}=\frac{1}{K} \sum_{i=1}^{K} E_{i}^{(\mathrm{ME})},
$$

where the term $E_{i}^{(\mathrm{ME})}$ is defined in (4) using the radio power levels as obtained by Algorithm (10). The total energy consumption (21) is non-decreasing with $\mathbf{P}$, since so does $f_{i}\left(P_{i}\right)$ in (4).

Considering the energy model for a system using ME coding (4), setting $\alpha_{\mathrm{ME}}=1$, and computing the radio powers with such an $\alpha$, we obtain the energy consumption of the BPSK case. The energy gain of the ME coding with respect to BPSK is defined as the ratio in $\mathrm{dB}$ of the energy used in a BPSK system and (21)

$$
\rho_{\mathrm{ME}}=10 \log \frac{E^{(\mathrm{BPSK})}}{E^{(\mathrm{ME})}} .
$$

\section{B. MME Coding}

To compute the energy needed by MME coding, it is necessary to characterize $T^{(\mathrm{rx}, \mathrm{MME})}$ and $N_{i}$ in (5). The older is given by the average time the receiver is in the active state per MME codeword times the bit time:

$$
T^{(\mathrm{rx}, \mathrm{MME})}=\bar{n}_{i} T_{b} .
$$

We have the following results

Proposition 2: The average time the receiver is in the active state per MME codeword is

$$
\begin{aligned}
\bar{n}_{i} & =N_{s}\left\{1+\operatorname{Pr}\left[b_{\text {ind }}=0\right]\left(1-e_{i \mid 0}\right)\left(L_{s}-1\right)\right. \\
& \left.+\operatorname{Pr}\left[b_{\text {ind }}=1\right] e_{i \mid 1}\left(L_{s}-1\right)\right\} .
\end{aligned}
$$

Proof: Since the sub-frame structure of an MME codeword, the receiver wakes up $N_{s}$ times to check the indicator bit. If the indicator bit is low and it is decoded as such, the receiver stays awake for the overall sub-frame, i.e., for 
$L_{s}-1$ bit. If the indicator bit is high, but it is erroneously decoded as a low one, again the receiver stays awake for the overall sub-frame. Adding up all the contributions, we obtain the expression.

Proposition 3: The average number of times that the radio module of the receiver goes from off to on is

$$
N_{i}=N_{s}\left(\operatorname{Pr}\left[b_{\text {ind }}=0\right] e_{i \mid 0}+\operatorname{Pr}\left[b_{\text {ind }}=1\right]\left(1-e_{i \mid 1}\right)\right) .
$$

Proof: The number of times that a receiver awakes can be computed from the number of times the receiver goes from the "on" to the "off" state. Indeed, each time the receiver turns off, it must turn on to decode the following indicator bit. The receiver is turned off each time an indicator bit is detected as high. For each of the $N_{s}$ sub-frames, this happens with probability $\operatorname{Pr}\left[b_{\text {ind }}=0\right] e_{i \mid 0}+\operatorname{Pr}\left[b_{\text {ind }}=1\right]\left(1-e_{i \mid 1}\right)$, from which (24) is obtained.

Eq. (23) and Eq. (24) can be used with (5) to obtain the energy consumption for the generic link $i$. Thus, averaging over all the links, the MME energy is:

$$
E^{(\mathrm{MME})}=\frac{1}{K} \sum_{i=1}^{K} E_{i}^{(\mathrm{MME})} .
$$

The total energy consumption (25) is non-decreasing with $\mathbf{P}$, since so do $f_{i}\left(P_{i}\right)$ and $T^{(\mathrm{rx}, \mathrm{MME})}+\left(N_{i}+1\right) T_{s}$.

Finally, the MME energy gain is defined as follows:

$$
\rho_{\mathrm{MME}}=10 \log \frac{E^{(\mathrm{ME})}}{E^{(\mathrm{MME})}} .
$$

\section{NUMERICAL RESULTS}

In this section, we provide numerical evaluation of the bit error probability and the total energy consumption of ME and MME coding. Analytical results, as obtained from the analysis carried out in the previous sections, are compared with those obtained from simulations.

The results have been carried out taking as reference the Tmote Sky wireless sensor nodes [11], which features the CC2420 radio transceiver module by Chipcon [12]. These sensors make use of DS-CDMA with a bit rate of $1 / T_{b}=$ $250 \mathrm{Kbps}$, and a processing gain $G=8$. The simulation parameters, which are introduced in the sequel, are therefore consistent with this hardware platform. A system scenario with $K=8$ pairs of nodes is analyzed. Nodes are deployed over an area where the maximum distance between a source node and a destination node of a pair is randomly chosen between $2 \mathrm{~m}$ and $15 \mathrm{~m}$, and the maximum distance between the transmitter of an interfering pair and a receiver is $15 \mathrm{~m}$. This choice is the most general and interesting, because each pair has a different distance source-destination, and each receiver will experience different interference, so that all the cases are accounted for. No larger distances are allowed, since this would require levels of radio power larger than $0 \mathrm{dBm}$, which is not possible with the Tmote Sky wireless sensor nodes. For each pair $i, j=1 \ldots K$, the standard deviation of the shadowing $\sigma_{\xi_{j i}}$ is randomly selected between $2 \mathrm{~dB}$ and $4 \mathrm{~dB}$. We assumed Raleigh fading, i.e., $m=1$. The power of the noise is set to $N_{0}=$ $-130 \mathrm{dBm}$. The values of the hardware energy consumption are $P^{(\mathrm{tx}, \mathrm{ckt})}=36 \times 10^{-3} \mathrm{~W}, P^{(\mathrm{rx}, \mathrm{ckt})}=33.84 \times 10^{-3} \mathrm{~W}$, and $P^{(\mathrm{rx}, \mathrm{ckt}, \mathrm{st})}=0 \mathrm{~W}$. Furthermore, $f\left(P_{i}\right)=V C\left(P_{i}\right)$, where

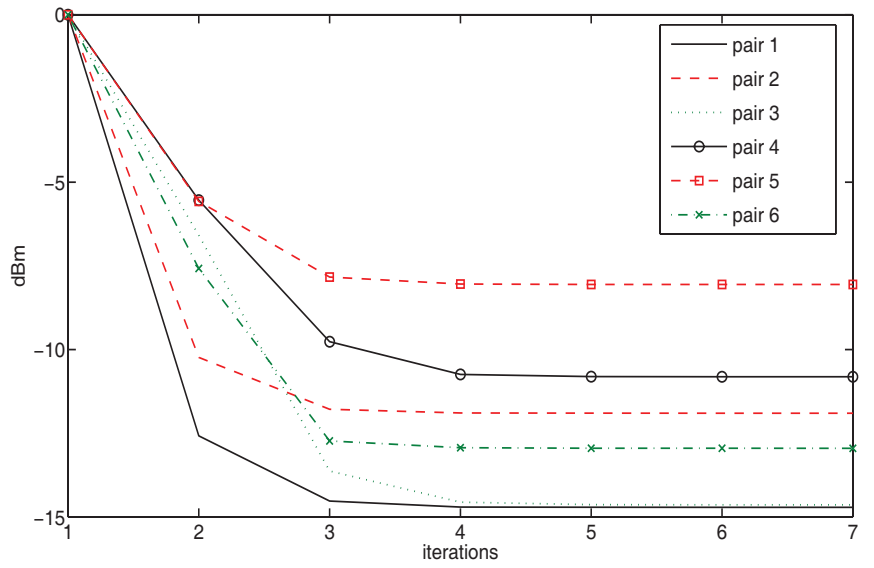

Fig. 5. Convergence of the power minimization algorithm, $\alpha=0.19$, $\bar{P}_{\text {out }}=0.01 \gamma=1$. On the $\mathrm{x}$-axis the number of iterations is reported.

$V=1.8 \mathrm{~V}$ is the voltage consumption and $C\left(P_{i}\right)$ is the current consumption of the electronic circuit needed to transmit with radio power $P_{i}$. The following relation can be derived [17]:

$$
\begin{aligned}
C\left(P_{i}\right)= & 6.4 \cdot 10^{-6} \log ^{3}\left(\frac{P_{i}}{10^{-3}}\right)+4.2 \cdot 10^{-3} \log ^{2}\left(\frac{P_{i}}{10^{-3}}\right) \\
& +9.9 \cdot 10^{-3} \cdot\left[\log \left(\frac{P_{i}}{10^{-3}}\right)+1.8\right] .
\end{aligned}
$$

We set the start-up time to $T_{s}=10 T_{b}$, a reasonable figure. Values of $T_{s} \leq 10 T_{b}$ provide the same results, as those shown below. Note that the start-up time of the Chipcon radio module [12] is $0.58 \times 10^{-3} \mathrm{~s}$. This case is however not that interesting to study because this long start-up time is not realistic for ME and MME coding. If numerical results were obtained with this figure, no advantage of MME with respect to ME would be achieved no matter what system parameters (fading, number of nodes, coding activity, codeword length) are, because this time is very long when compared to the bit time. Furthermore, such a high wake up time would put a severe limitation to packet transmission, since no bit reception is possible during that long time.

In Fig. 5, the convergence trace of the power control Algorithm (10) is reported for the case of $\bar{P}_{\text {out }}=0.01$, $\alpha_{\mathrm{ME}}=0.19$, and $\gamma=1$. Each curve refers to the radio power of a pair. Convergence is fast, with less than 5 iterations. This behavior remains the same for other choices of the system parameters.

In the sequel, we present numerical results for two representative cases: short codewords $\left(N_{s}=3, L_{s}=20\right.$, Figs. $\left.6-8\right)$, and long codewords $\left(N_{s}=20, L_{s}=20\right.$, Figs. $\left.9-12\right)$. These parameters were chosen considering that the longest codeword allowed for the payload with the Tmote Sky sensor nodes is 760 bit.

In Fig. 6, the ME energy gain as obtained by (22) in the case of $N_{s}=3$ and $L_{s}=20$ is plotted versus the SINR threshold $\gamma$. Each curve refers to a different value of the coding $\alpha_{\mathrm{ME}}$, as obtained after the convergence of the radio power control Algorithm (10). It is evident that the ME coding introduces significant energy savings, in particular for low values of $\alpha_{\mathrm{ME}}$. Surprisingly, as $\gamma$ tends to 1 , the gain quickly decreases. This is due to the fact that large values of $\gamma$ require higher radio 


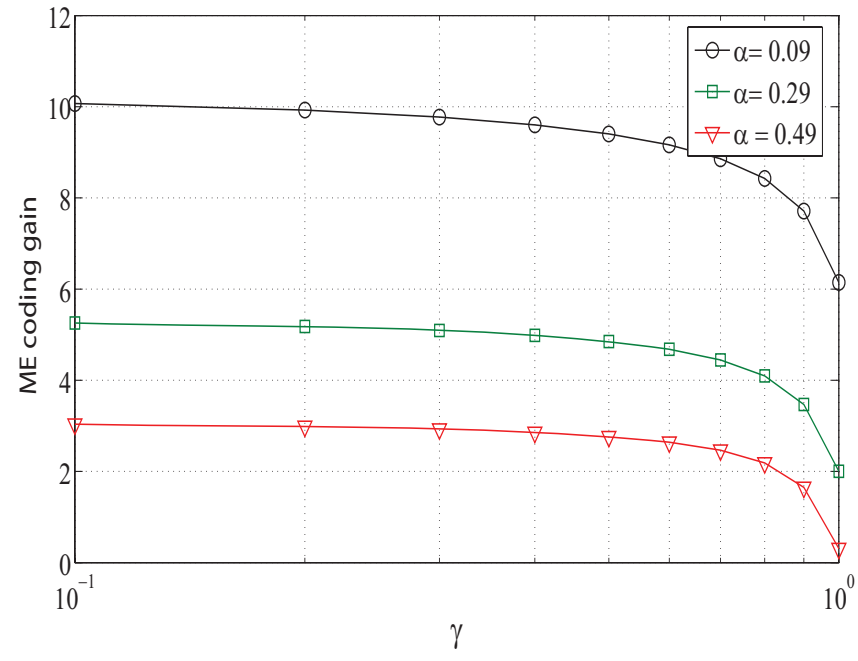

Fig. 6. ME Energy Gain as function of the SINR threshold $\gamma$ for different values of $\alpha_{\mathrm{ME}}=\alpha, \bar{P}_{\text {out }}=0.01, N_{s}=3$ and $L_{s}=20$.

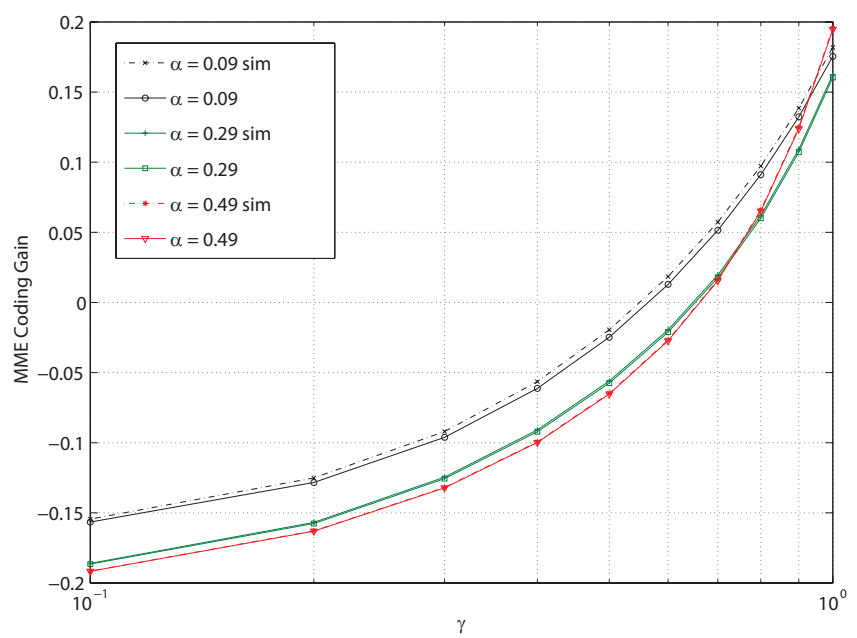

Fig. 7. MME Energy Gain as function of the SINR threshold $\gamma$ for different values of $\alpha_{\mathrm{MME}}=\alpha, \bar{P}_{\text {out }}=0.01, N_{s}=3$ and $L_{s}=20$.

powers, with the consequence that the energy spent for radio power transmission $\alpha_{\mathrm{ME}} f\left(P_{i}\right)$ assumes large values when compared to the energy spent by the electronic circuits while transmitting and processing a codeword. No value of $\gamma$ larger than 1 is admitted, because the outage constraint cannot be guaranteed, i.e., for $\gamma>1$ there may be at least a pair in outage.

In Fig. 7, the MME energy gain as obtained by (26) in the case of $N_{s}=3$ and $L_{s}=20$ is plotted versus the SINR threshold $\gamma$. Each curve refers to a different value of the coding $\alpha_{\mathrm{MME}}$, as obtained after the convergence of the Algorithm (10). MME coding does not show any advantage with respect to ME when $\gamma<0.7$. Indeed, low values of $\gamma$ imply large value of the MME bit error probability, which causes the receiver to commit frequent errors when decoding of the indicator bit. The consequence is that the receiver is awake even though it was not necessary. Furthermore, recalling that the MME energy balance (5) includes the extra

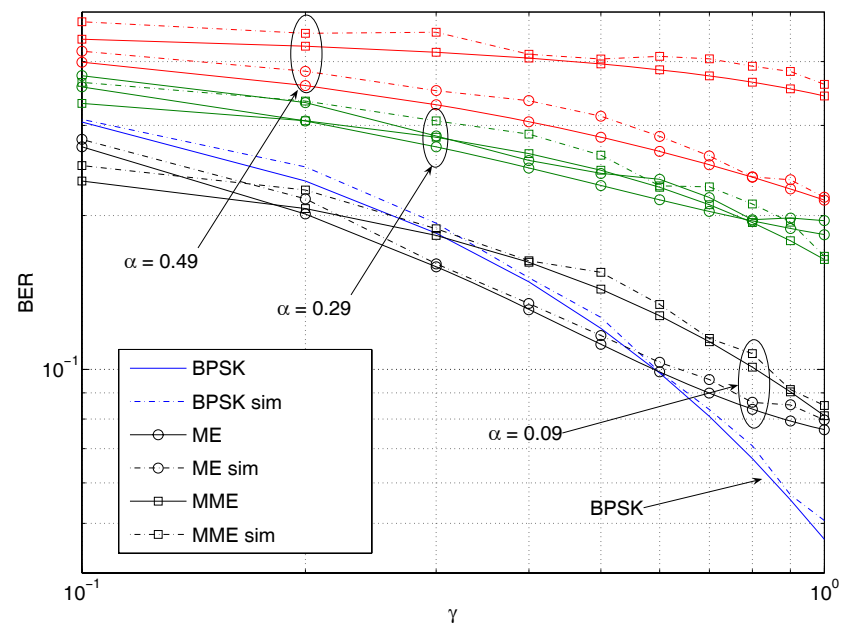

Fig. 8. BER for BPSK, ME and MME as function of the SINR threshold $\gamma$ for different values of $\alpha_{\mathrm{ME}}=\alpha_{\mathrm{MME}}=\alpha, \bar{P}_{\mathrm{out}}=0.01, N_{s}=3$ and $L_{s}=20$.

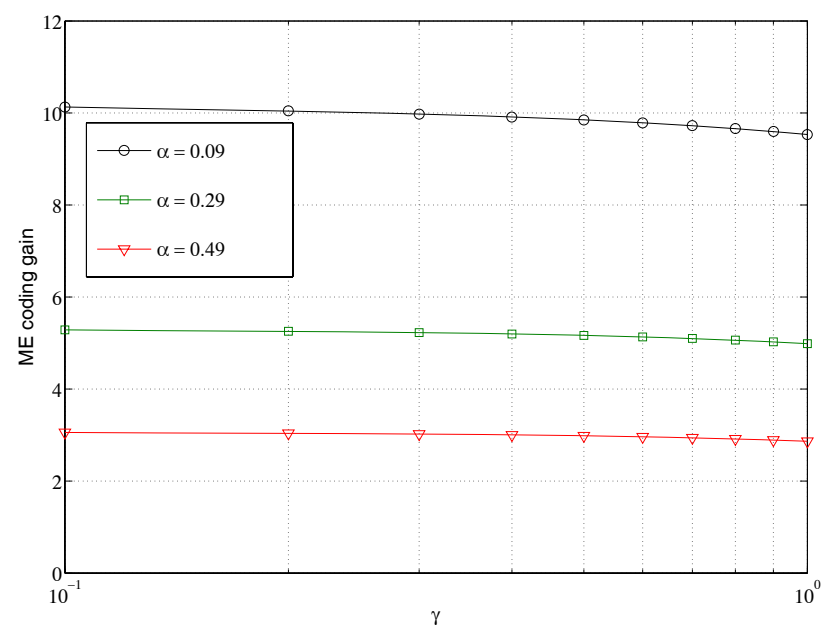

Fig. 9. ME Energy Gain as function of the SINR threshold $\gamma$ for different values of $\alpha_{\mathrm{ME}}=\alpha, \bar{P}_{\text {out }}=0.01, N_{s}=20$ and $L_{s}=20$.

term $N_{i}$ with respect to the ME energy consumption (4), this explains the poor performance of MME when $\gamma \leq 0.7$. On the contrary, when $\gamma \geq 0.7$, the ME bit error probability decreases, so that the MME receiver goes often to sleep, and $N_{i}$ is also reduced. The MME gain increases as the the coding activity increases. Indeed, the ME coding gain worsens in such circumstances, while the MME is able to save energy by going to sleep. This is an interesting result, since it means that for large $\gamma$ it is better to use short codes (i.e., codes with larger activity), rather then longer codes.

In Fig. 8, the bit error probability in the case of $N_{s}=3$, and $L_{s}=20$ is reported for BPSK, ME and MME coding versus the SINR threshold $\gamma$ as obtained in section V-A and V-B. Each curve is associated to a different value of the coding activity, as obtained after the convergence of the radio power control Algorithm (10). There is no common pattern in the behavior of the bit error rate with respect to ME and MME 


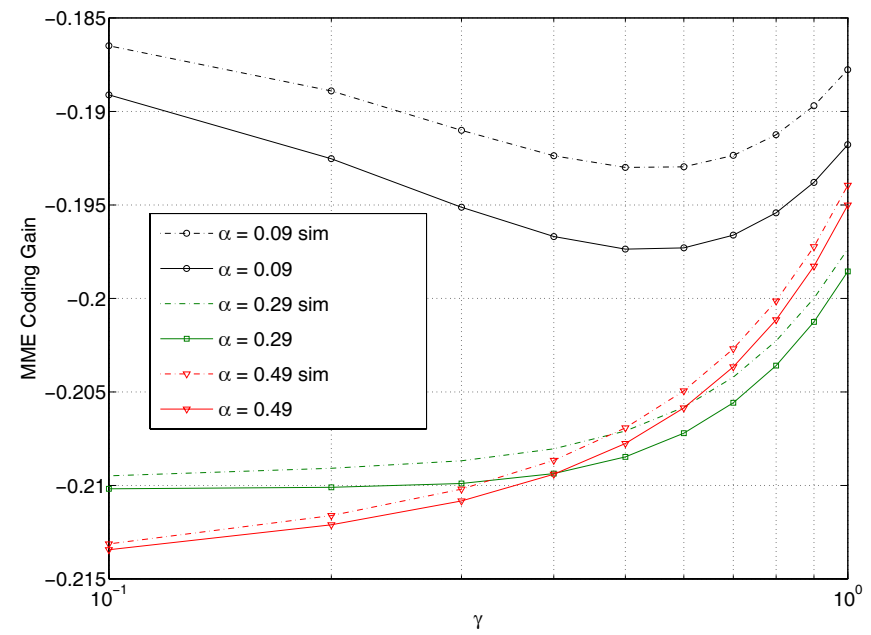

Fig. 10. MME Energy Gain as function of the SINR threshold $\gamma$ for different values of $\alpha_{\mathrm{MME}}=\alpha, \bar{P}_{\mathrm{out}}=0.01, N_{s}=20$ and $L_{s}=20$.

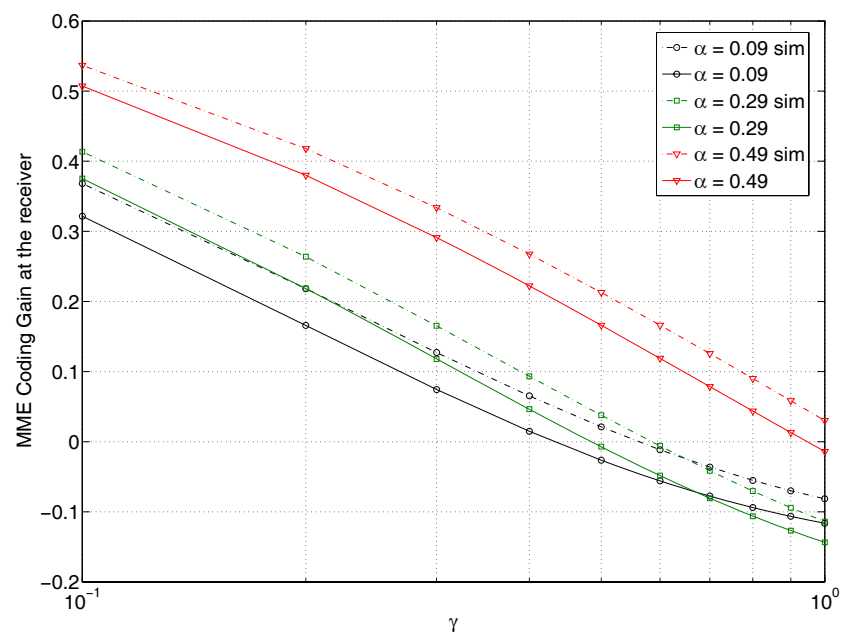

Fig. 11. MME Energy Gain at the receiver as function of the SINR threshold $\gamma$ for different values of $\alpha_{\mathrm{MME}}=\alpha, \bar{P}_{\text {out }}=0.01, N_{s}=20$ and $L_{s}=20$.

coding. The BER of BPSK is lower then the BER of ME and MME for $\gamma \geq 0.6$. This is an interesting result, since one would expect that the OOK modulation increases the BER. However, ME and MME cause less interference, which explains their better performance when $\gamma<0.5$. By the same argument, increasing the coding activity leads often to an increase of the BER of ME and MME. However, notice that small $\gamma$ and large $\alpha_{\mathrm{MME}}$ yield the worst case of the BER (recall that this choice of parameters gives the best results in terms of energy).

Fig. 9 presents the ME energy gain as obtained by (22) in the case of $N_{s}=20$ and $L_{s}=20$ versus the SINR threshold $\gamma$. The main difference with respect to the short codeword case in Fig. 6 is that the gain does not decrease substantially when $\gamma$ increases. This is because a longer codeword rises the importance of the terms $P^{(\mathrm{tx}, \mathrm{ckt})}$ and $P^{(\mathrm{rx}, \mathrm{ckt})}$ in (22) and (26), respectively, so that the energy for radio power is less relevant with respect to the case of short codewords.

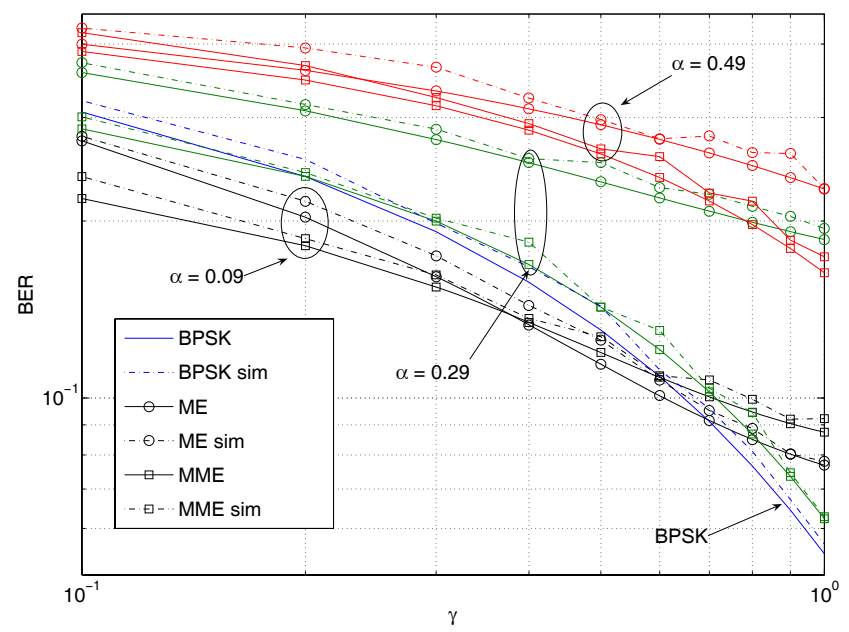

Fig. 12. BER for BPSK, ME and MME as function of the SINR threshold $\gamma$ for different values of $\alpha_{\mathrm{ME}}=\alpha_{\mathrm{MME}}=\alpha, \bar{P}_{\mathrm{out}}=0.01, N_{s}=20$ and $L_{s}=20$.

In Fig. 10, the MME energy gain as obtained by (26) in the case of $N_{s}=20$ and $L_{s}=20$ is plotted versus the SINR threshold $\gamma$. Each curve refers to a different value of the coding $\alpha_{\mathrm{MME}}$. Observe that MME coding does not show any advantage with respect to ME for any value of $\gamma$. Choosing large codewords means to increase substantially the term $T^{(\mathrm{tx}, \mathrm{MME})}=N_{s} L_{s} T_{b}$ in (26), whereas notice that $T^{(\mathrm{tx}, \mathrm{ME})}=N_{s}\left(L_{s}-1\right) T_{b}$. The time spent to transmit is responsible also for the energy spent for radio power transmission. This explains the poor performance of MME. However, MME is still able to save energy at the receiver node, as shown in Fig. (11). This gain is defined similarly to (26), but using only the energy $E_{i}^{(\mathrm{rx})}$ in (4) for $\mathrm{ME}$, and $E_{i}^{(\mathrm{rx})}$ in (5) for MME. The receiver MME energy gain decreases with $\gamma$, since so does the number of starts-up $N_{i}$. The major consequence that can be inferred from Fig. 7 and Fig. 10 is that MME does not allow for global energy savings when using large codewords.

Finally, In Fig. 12, the bit error probability in the case of $N_{s}=20$ and $L_{s}=20$ is reported for BPSK, ME, and MME coding versus the SINR threshold $\gamma$. The same considerations made for Fig. 8 still hold. The main difference is that there is just a slight increase of the BER, as obvious consequence of longer codewords.

\section{CONCLUSIONS}

We presented a general framework for the comparison of ME and MME coding in CDMA-based wireless sensor networks. The analysis took into account radio power consumption, energy consumption of the electronic circuit transceivers, and bit error probability. A distributed minimization of the total energy consumption was proposed, and a novel detection threshold method for OOK modulation was suggested.

Numerical results show that ME coding always outperforms BPSK in terms of energy consumption, and, for certain regions of outage and coding activity, also in terms of bit error rate. MME coding outperforms ME only for small-size codewords, 
while it shows poor performance for large codewords, because of the significant energy spent for start-up. However, as technology evolves and smaller start-up times and energy are possible, MME may be favorable also with longer codewords.

\section{REFERENCES}

[1] C. Erin and H. H. Asada, "Energy optimal codes for wireless communications," in Proc. 38th IEEE Conf. of Decision and Control, 1999.

[2] C. Erin, H. H. Asada, and K.-Y. Siu, "Minimum energy coding for RF transmission," in Proc. IEEE Wireless Commun. Networking Conf., 1999.

[3] C. Fischione, A. Bonivento, A. Sangiovanni-Vincentelli, F. Santucci, and K. H. Johansson, "Performance analysis of collaborative spatio-temporal processing for wireless sensor networks," in Proc. IEEE Consumer Commun. Conf. 2006, Jan. 2006.

[4] J. Kim and J. G. Andrews, "An energy efficient source coding and modulation scheme for wireless sensor networks," in Proc. 6th IEEE Workshop Signal Processing Advances Wireless Commun., 2005.

[5] Y. Prakash and S. K. Gupta, "Energy efficient source coding and modulation for wireless applications," in Proc. IEEE Wireless Commun. Networking Conf., 2003.

[6] Q. Tang, S. Gupta, and L. Schwiebert, "BER performance analysis of an on-off keying based minimum energy coding for energy constrained wireless sensor application," in Proc. IEEE International Conf. Commun., 2005.

[7] C. H. Liu and H. H. Asada, "A source coding and modulation method for power saving and interference reduction in DS-CDMA sensor networks systems," in Proc. Americal Control Conf., 2002.

[8] B. Zurita Ares, C. Fischione, and K. H. Johansson, "Energy consumption of minimum energy coding in CDMA wireless sensor networks," in Proc. 4th European Conf. Sensor Networks 2007, Jan. 2007.

[9] F. Santucci, G. Durastante, F. Graziosi, and C. Fischione, "Power allocation and control in multimedia CDMA wireless systems," Kluwer Telecommun. Syst., vol. 23, pp. 69-94, May/June 2003.

[10] G. L. Stüber, Principles of Mobile Communication. Kluwer Academic Publishers, 1996.

[11] "Moteiv," San Francisco, CA, Tmote Sky Data Sheet, 2006.

[12] Chipcon Products, "2.4 GHz IEEE 802.15.4 zigbee-ready RF transceiver," technical report, Texas Instruments, 2006.

[13] C. Fischione, F. Graziosi, and F. Santucci, "Approximation for a sum of on-off log-normal processes with wireless applications," IEEE Trans. Commun., vol. 55, no. 10, pp. 1984-1993, Oct. 2007.

[14] C. Fischione, M. Butussi, K. H. Johansson, and M. D'Angelo, "Power and rate control with outage constraints in CDMA wireless networks," IEEE Trans. Commun., 2008, to appear.

[15] D. P. Bertsekas and J. N. Tsitsiklis, Parallel and Distribuited Computation: Numerical Methods. Athena Scientific, 1997.

[16] S. Boyd and L. Vandenberghe, Convex Optimization. Cambridge University Press, 2004.

[17] D. Lymberopoulos and A. Savvides. "XYZ: a motion-enabled, power aware sensor node platform for distributed sensor network applications," in Proc. Information Processing Sensor Networks (IPSN) 2005.

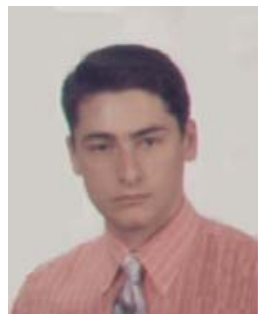

Carlo Fischione received the Ph.D. degree in Electrical and Information Engineering and the Laurea degree in Electronic Engineering (summa cum Laude) in May 2005 and April 2001, respectively, both from the University of L'Aquila, Italy. Since May 2008 he is with the Royal Institute of Technology, ACCESS Linnaeus Centre, Electrical Engineering, Stockholm, Sweden, as an Assistant Professor. $\mathrm{He}$ was a postdoctoral research associate at the University of California at Berkeley, Department of Electrical Engineering and Computer Sciences (May 2007 - September 2008), and a postdoctoral research associate at the Automatic Control Group, Royal Institute of Technology (May 2005 - May 2007). He received the best paper award for the IEEE TRANSACTIONS ON INDUSTRIAL INFORMATICS during the year 2007, a best paper award of the IEEE International Conference on Mobile Ad-hoc and Sensor System 05 (IEEE MASS 05), the Ferdinando Filauro award from the University of L'Aquila, Italy, and the Alta Formazione award of the Abruzzo Region Government, Italy. His research interests are in the area of wireless networks and networked embedded systems, with particular reference to wireless communication theory, control of wireless networks, and optimization. $\mathrm{He}$ is a member of the IEEE Communications Society and serves as reviewer for technical journals and conferences.

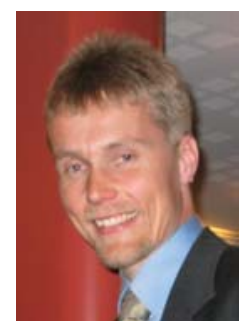

Karl H. Johansson received an M.Sc. and a Ph.D. in Electrical Engineering in 1992 and 1997, respectively, both from Lund University in Sweden. He is currently Professor and Co-director of the ACCESS Linnaeus Centre at the School of Electrical Engineering, Royal Institute of Technology, Sweden. He holds a Senior Researcher Position at the Swedish Research Council. He has held visiting positions at UC Berkeley (1998-2000) and California Institute of Technology (2006-07). His research interests are in networked control systems, hybrid and embedded control, and control applications in automotive, automation and communication systems. He is the Chair of the International Federation of Automatic Control (IFAC) Technical Committee on Networked Systems since 2008. He has served on the Executive Committees of the European research projects HYCON and RUNES, both on networked embedded systems. He is on the editorial boards of IEEE TRANSACTIONS ON AUTOMATIC CONTROL and IET CONTROL THEORY \& APPLICATIONS, and previously of AUTOMATICA. $\mathrm{He}$ was awarded an Individual Grant for the Advancement of Research Leaders from the Swedish Foundation for Strategic Research in 2005. He received the triennial Young Author Prize from IFAC in 1996 and the Peccei Award from the International Institute of System Analysis, Austria, in 1993. He received Young Researcher Awards from Scania in 1996 and from Ericsson in 1998 and 1999.

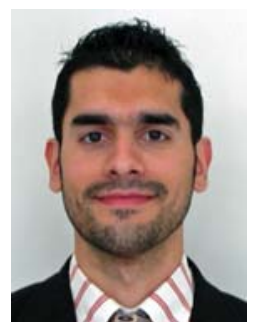

Benigno Zurita Ares received the M.Sc. in Telecommunications Engineering from University of Seville, Spain, in 2006. He developed his M.Sc. Thesis at the Royal Institute of Technology, Stockholm, Sweden in 2006, where he was an assistant researcher at the Automatic Control Group, School of Electrical Engineering. His research interests are in the area of networked control systems, embedded control and communication systems, focusing mainly on wireless sensor networks. Since 2007 , he is working in an aerospace and military company, developing boarded systems.

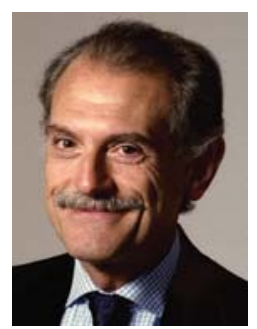

Alberto Sangiovanni-Vincentelli holds the Edgar L. and Harold H. Buttner Chair of Electrical Engineering and Computer Sciences at the University of California at Berkeley where he has been on the Faculty since 1976. In 1980-1981, he spent a year as a Visiting Scientist at the Mathematical Sciences Department of the IBM T.J. Watson Research Center. In 1987, he was Visiting Professor at MIT.

$\mathrm{He}$ was a co-founder of Cadence and Synopsys, the two leading companies in the area of Electronic Design Automation. He is the Chief Technology Advisor of Cadence Design System. He is a member of the Board of Directors of Cadence, Sonics Inc., UPEK, Value Partners and Accent. He was a member of the HP Strategic Technology Advisory Board. He is a member of the GM Science and Technology Advisory Board, and the founder and Scientific Director of PARADES, a European Group of Economic Interest supported by Cadence and ST Microelectronics. He is a member of the High-Level Group and of the Steering Committee of the EU Artemis Technology Platform.

In 1981 he received the Distinguished Teaching Award of the University of California. He received the worldwide 1995 Graduate Teaching Award of the IEEE for "inspirational teaching of graduate students." He has received numerous best paper awards including the Guillemin-Cauer Award (19821983) and the Darlington Award (1987-1988). In 2001, he was given the prestigious Kaufman Award of the Electronic Design Automation Council for pioneering contributions to EDA. In 2002, he was the recipient of the Aristotle Award of the Semiconductor Research Corporation. He was elected Fellow of the IEEE in 1982 and to the National Academy of Engineering in 1998. He is an author of over 800 papers and 15 books in the area of design tools and methodologies, large-scale systems, embedded controllers, hybrid systems and innovation. 